\title{
Spontaneous emission and thermalization of cold bosons in optical lattices
}

\author{
J. Schachenmayer, ${ }^{1}$ L. Pollet, ${ }^{2}$ M. Troyer, ${ }^{3}$ and A. J. Daley ${ }^{1}$ \\ ${ }^{1}$ Department of Physics and Astronomy, University of Pittsburgh, Pittsburgh, Pennsylvania 15260, USA \\ ${ }^{2}$ Department of Physics, Arnold Sommerfeld Center for Theoretical Physics and Center for NanoScience, \\ University of Munich, 80333 Munich, Germany \\ ${ }^{3}$ Theoretische Physik, ETH Zurich, 8093 Zurich, Switzerland
}

(Received 6 May 2013; published 10 January 2014)

\begin{abstract}
We study the thermalization of excitations generated by spontaneous emission events for cold bosons in an optical lattice. Computing the dynamics described by the many-body master equation, we characterize equilibration time scales in different parameter regimes. For simple observables, we find regimes in which the system relaxes rapidly to values in agreement with a thermal distribution, and others where thermalization does not occur on typical experimental time scales. Because spontaneous emissions lead effectively to a local quantum quench, this behavior is strongly dependent on the low-energy spectrum of the Hamiltonian, and undergoes a qualitative change at the Mott insulator-superfluid transition point. These results have important implications for the understanding of thermalization after localized quenches in isolated quantum gases, as well as the characterization of heating in experiments.
\end{abstract}

Spontaneous emission is a fundamental source of heating in optical dipole potentials [1,2], and one of the key heating sources in current experiments with cold atoms in optical lattices $[3,4]$. This heating induces nonequilibrium dynamics in which thermalization processes are expected to play a key role. Typically it is assumed that the energy added to the system will be thermalized, causing an effective increase in temperature. But does that happen?

This question is a special case of a fundamental problem in many-body quantum mechanics: To what extent, and under which conditions, will an isolated system undergo thermalization when perturbed away from equilibrium, in the sense that at long times the system reaches a steady state where simple observables take the same values as those of a thermal distribution [5-8]. Recently, experiments with strongly interacting cold gases confined to move in one dimension (1D) [9] have demonstrated regimes of integrable dynamics-where systems do not thermalize in a traditional sense [10], although they can sometimes relax to a steadystate distribution described by a generalized Gibbs ensemble $[11,12]$.

In this Rapid Communication we investigate these issues by studying dynamics induced by spontaneous emissions (incoherent light scattering) for cold bosons in an optical lattice [13], and identify contrasting parameter regimes where (i) certain observables relax over short times to thermal values, or (ii) the system relaxes on a short time scale to states that are clearly nonthermal. The dynamics depends greatly on the low-energy spectrum of the Hamiltonian because spontaneous emissions give rise to a local quench, leading to qualitative changes at the superfluid-Mott insulator phase transition. By combining time-dependent density matrix renormalization group (t-DMRG) methods [14-17] with quantum trajectory techniques [18-20], we compute the dynamics in the context of real experiments. These results have important implications for the characterization of heating in current experiments [21]. In fact, the lack of thermalization of certain excitations may be exploited to enhance the realization of fragile many-body states [22-25], leading to greater robustness of quantum simulators $[26,27]$. Below we first summarize the effects of spontaneous emissions on atoms in an optical lattice, before analyzing thermalization in the lowest Bloch band.

Effect of spontaneous emissions. The scattering of a photon via spontaneous emission effectively provides the environment with information about the position of an atom [1,2]. This leads to two key physical effects on bosons beginning in the lowest band of an optical lattice [3,4]: It can (i) transfer atoms to higher bands, and/or (ii) localize atoms on the length scale of the photon wavelength $\lambda$.

Transfer of atoms to higher bands is suppressed for the relatively deep optical lattices found in experiments by the square of the Lamb-Dicke parameter, $\eta=2 \pi a_{T} / \lambda$, where $a_{T}$ is the trap length for the lowest band Wannier function. For typical experiments with lattice depths around $V_{0}=8 E_{R}$ [with $E_{R}=4 \pi^{2} \hbar^{2} /\left(2 m \lambda^{2}\right)$, where $m$ is the mass of the atom], $\eta^{2} \sim$ 0.1 , and if the lattice is red-detuned, the dominant dissipative processes return atoms to the lowest band. These rare band transfer processes give rise to a large energy increase of the order of the band-gap energy $\omega_{g}(\hbar \equiv 1)$. This energy is much larger than energy scales in the lowest band, which prevents thermalization of $\omega_{g}$ on experimental time scales because it would require a collision with many atoms simultaneously to transfer the energy to the lowest band. ${ }^{1}$ This is analogous to the collisional stability of doublon pairs demonstrated in recent experiments [28].

Heating and thermalization in the lowest band. For processes where the atom remains in the lowest band, this question is substantially more complicated. A spontaneous emission localizes the atoms on the scale of a single site [4], because the wavelength is comparable to the lattice spacing $\lambda / 2 \sim a$. This is in contrast with photon scattering

\footnotetext{
${ }^{1}$ Note that collisional processes between two or more atoms in the first excited band can return particles to the lowest band while exciting atoms to higher bands. This does not affect the conclusion that the band-gap energy cannot be thermalized with the atoms in the lowest band.
} 
in solid state physics, where $\lambda$ is much larger than the lattice spacing. These processes increase the energy on scales of the width of the lowest band, as atoms are transferred to higher quasimomentum states.

Thermalization properties then depend on dynamics described by the Bose-Hubbard model,

$$
H=-J \sum_{\langle\mathbf{i}, \mathbf{j}\rangle} b_{i}^{\dagger} b_{j}+\frac{U}{2} \sum_{\mathbf{i}} b_{i}^{\dagger} b_{i}^{\dagger} b_{i} b_{i}+\sum_{\mathbf{i}} \varepsilon_{\mathbf{i}} b_{i}^{\dagger} b_{i} .
$$

Here, $b_{i}^{\dagger}$ is a bosonic creation operator for an atom on site $\boldsymbol{i}, J$ denotes the tunneling rate between neighboring sites, $U$ is the on-site interaction, and $\varepsilon_{\mathbf{i}}$ is the on-site potential. This model is nonintegrable outside the limiting cases of $U \rightarrow 0$ and $U / J \rightarrow \infty$, and has been shown to exhibit chaotic spectral properties when $U \sim J$ [29,30]. As a result, it might be expected that the system will thermalize for most values of $U / J$, with the most rapid thermalization around $U \sim J$. For high values of $U / J$, the system behaves as hard-core bosons, relaxing to a generalized Gibbs ensemble [10,31]. This is what is typically expected for a global quench of the value of $U / J$ [32]. However, it is not clear that this analysis applies to our situation because a spontaneous emission event leads to localization of atoms in a local quantum quench with excitations that are very low in energy. Because the lowest part of the energy spectrum can exhibit spectral statistics closer to an integrable model [29], this may even result in a lack of thermalization for all values of $U / J$. Below we find that the relaxation time scales and equilibrium values strongly depend on the interactions in the lower band (as it is also observed for local quenches in 2D [33-35]).

In the lowest band, the heating and thermalization together can be effectively described by a master equation [4] (see Supplemental Material [36]),

$$
\dot{\rho}=-i[H, \rho]-\frac{\gamma}{2} \sum_{\mathbf{i}}\left[\hat{n}_{i},\left[\hat{n}_{i}, \rho\right]\right],
$$

where $H$ is the Bose-Hubbard Hamiltonian (1). The dissipative dynamics involve localization of particles on a single site via scattering of photons at a rate $\gamma$, which depends on the intensity of the lattice lasers and the detuning from resonance.

Thermalization after a single intraband spontaneous emission event. In order to characterize the thermalization process, we first consider the situation where the system is in the ground state of model (1) $\left|\psi_{g}\right\rangle$ at time $t=0$, and undergoes a spontaneous emission (on site $i$ ). In the sense of continuous measurement theory [19] applied to (2), the resulting state prepared is

$$
\left|\psi_{\mathbf{i}}\left(t=0^{+}\right)\right\rangle=\frac{\hat{n}_{\mathbf{i}}\left|\psi_{g}\right\rangle}{\| \hat{n}_{\mathbf{i}}\left|\psi_{g}\right\rangle \|}
$$

We consider a weighted ensemble average over the sites $\mathbf{i}$ with probabilities of spontaneous emission $p_{\mathbf{i}} \propto\left\langle\psi_{g}\left|n_{\mathbf{i}}^{2}\right| \psi_{g}\right\rangle$, and treat a 1D system, where we can use t-DMRG methods to propagate the state exactly in time. Note that all tDMRG results are converged in the matrix product state bond dimension $D$ and the truncation of the local dimension $d_{l}$.

Figure 1(b) shows the typical dynamics after a spontaneous emission spreads a particle over the whole Brillouin zone and increases the kinetic energy $E^{\mathrm{kin}}$. The interactions between

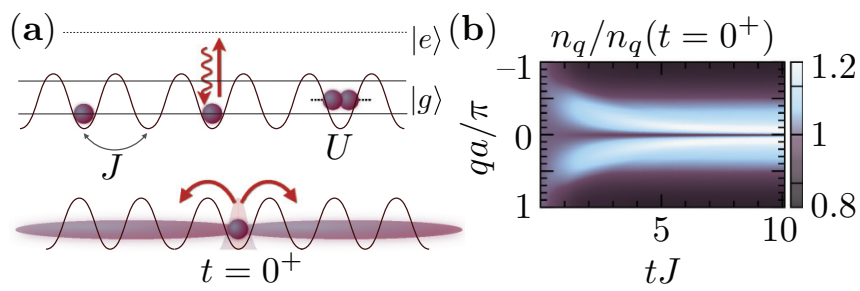

FIG. 1. (Color online) (a) Absorption and spontaneous emission of a lattice photon leads effectively to localization of single atoms. Tunneling and interactions between atoms then redistribute the energy added to the system. (b) Localization of an atom in space corresponds initially to a distribution of the atom over the whole Brillouin zone (the tails of the quasimomentum distribution are lifted). Subsequent unitary evolution leads to a broadened quasimomentum distribution, i.e., the $n_{q=0}$ peak and the tails decrease, while the small quasimomentum components increase (t-DMRG, $U=2 \mathrm{~J}, N=48$ particles on $M=48$ sites, $\left.d_{l}=6, D=512\right)$.

particles transfer some of this increased kinetic energy to interaction energy, as shown explicitly in Fig. 2(a) for an initial superfluid (SF) state with $U=2 J$. At $t=0^{+}, E^{\text {kin }}$ is increased by an amount of the order of $J$ over the ground-state value, and it then relaxes to a lower value over a time scale $\sim 5 / J$ in unitary time evolution. We obtain an equilibrium value $E_{\mathrm{eq}}^{\mathrm{kin}}$ from path integral Monte Carlo (QMC) calculations with worm-type updates [37] (here in the implementation of Ref. [38] - see Ref. [39] for a recent review of the method with applications to cold gases) at finite temperature $T$, fitting $T$ to match the value of energy $\langle E\rangle$ for $t \geqslant 0^{+}$. It is remarkable that this value corresponds to the equilibrium value reached dynamically within statistical errors, indicating thermalization of this quantity. In contrast, for an initial Mott insulator (MI, $U=4 J)$ state, $E^{\text {kin }}$ relaxes on a slightly longer time scale to an equilibrium value that clearly does not correspond to a thermal distribution at the appropriate value of $\langle E\rangle$. In fact, in this parameter regime, thermally induced coherence in the MI leads to a $E_{\text {eq }}^{\text {kin }}$ being close or even below the value of the ground-state kinetic energy [40].

In Fig. 2(c) we compare the extrapolated equilibrium kinetic energy $E_{\infty}^{\text {kin }}$ (obtained from an exponential fit) to $E_{\mathrm{eq}}^{\text {kin }}$ for various system sizes and interaction strengths. The lack of thermalization for values of $U / J$ immediately above the SF-MI transition point (when the gap is about $\Delta=J / 8$ ) is striking. Although from our calculations we cannot rule out a second relaxation process to a thermal distribution for much larger systems or on much longer time scales, it is clear that a qualitative change in behavior occurs here, leading to a lack of thermalization on typical experimental time scales. Before performing these calculations, we might have expected a crossover behavior, similar to that seen in the relaxation rates, as shown in Fig. 2(d) from exponential fits to the long-time behavior of $E^{\mathrm{kin}}$, where the fastest relaxation occurs for $U / J \sim 1$.

Note that as with thermalization in any closed quantum system, the behavior depends on the observable considered, and sufficiently complicated or nonlocal observables will never thermalize [5]. In Figs. 2(e) and 2(f), we show the quasimomentum distribution $n_{q}$ in our system with open 

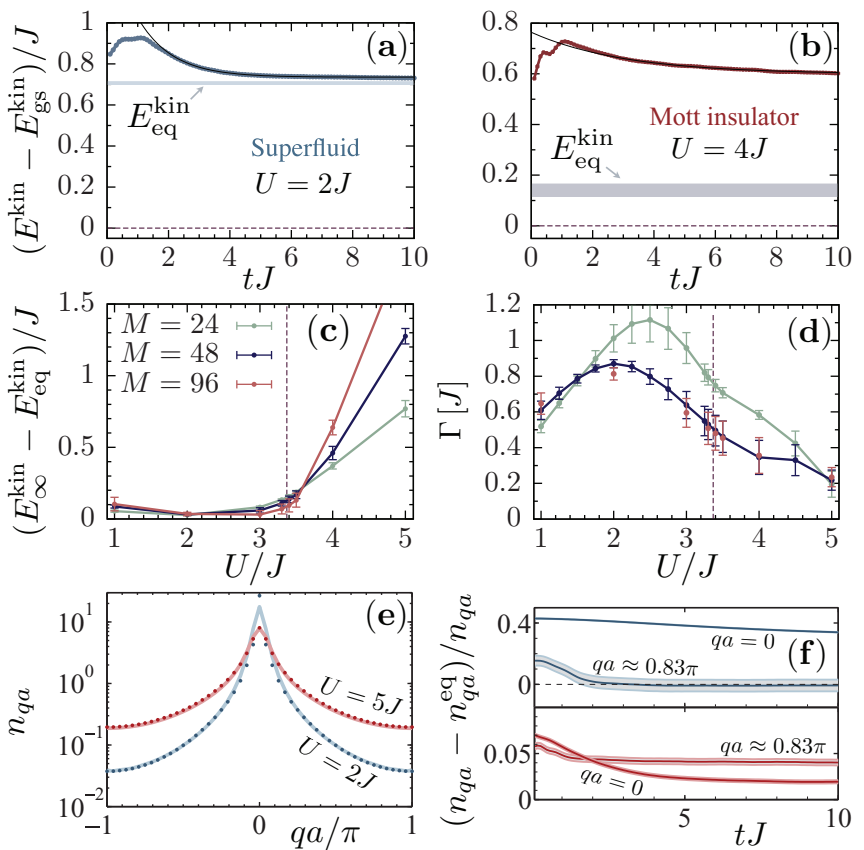

FIG. 2. (Color online) Time evolution after a single spontaneous emission. (a), (b) For a superfluid initial state $(U=2 J)$, the kinetic energy relaxes to the equilibrium value obtained from a Monte Carlo calculation $E_{\mathrm{eq}}^{\mathrm{kin}}$. For MI states $(U=4 J)$, the energy relaxes, but not to $E_{\mathrm{eq}}^{\mathrm{kin}}$. The zero value of kinetic energy for this plot is the ground-state kinetic energy $E_{\mathrm{gs}}^{\mathrm{kin}}$. (c) The difference in the infinite time value of the kinetic energy (obtained from an extrapolation of an exponential fit) to the equilibrium energy. For MI states with $U / J \gtrsim 3.37$, the difference increases rapidly for $M=24,48,96$ sites. (d) The decay rate extracted from the exponential fit as a function of $U$. (e) Comparison of the time-evolved quasimomentum distribution at $t=10 / J$ (dots) to the equilibrium distribution from a QMC calculation. (f), (g) Differences between the two distributions as a function of time for the $q a=0$ peak and for a large quasimomentum of $q a=(40 / 48) \pi$. In the superfluid $[U=2 J$ in (f)], the components for large momenta relax rapidly to thermal values, for $q a \sim 0$, the relaxation time scale is much longer. In the MI $[U=5 J$ in $(\mathrm{g})]$, the same is true, but for large momenta there is a discrepancy to the thermal value. (t-DMRG, $d_{l}=6, D=256,512$; error bars represent fitting errors and statistical errors from QMC.)

boundary conditions for different points in time. For all $q$ except very near $q=0, n_{q}$ relaxes to a thermal distribution on time scales $t J \sim 5$ in the SF for $U \gtrsim 1$. However, longwavelength modes around $n_{q=0}$ require much longer relaxation time scales, and are still far from their steady-state values on the time scales computed here (though they are evolving towards the expected thermal value). In the MI, the distribution behaves qualitatively differently, in that all values of $q$ show small discrepancies from the equivalent thermal values, consistent with what we observed for the kinetic energy. While these discrepancies are small for a single spontaneous emission event, they can be much larger when multiple photons are scattered in the experimental protocol discussed below.

Explanation based on the low-energy spectrum. The key to understanding the qualitative change in behavior at the transition point lies in the fact that the spontaneous emissions give rise to a local quantum quench, which only significantly populates low-energy eigenstates. Most of the amplitude of the
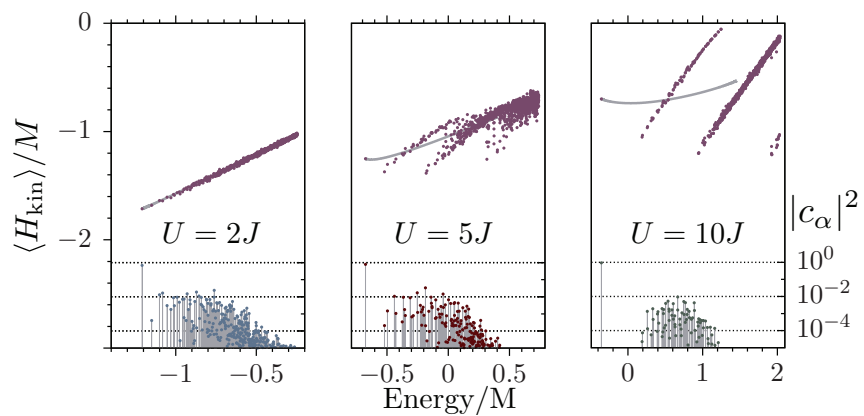

FIG. 3. (Color online) Expectation values of the kinetic energy of the lowest 1000 eigenstates as a function of the energy in a system with $M=10$ and $N=10$ (exact diagonalization). The gray line in the upper plot shows the equilibrium kinetic energy $E_{\text {eq }}^{\text {kin }}$ for increasing temperatures as a function of the mean energy of the underlying Boltzmann distributions. In the SF, the eigenvalue expectations are distributed around $E_{\mathrm{eq}}^{\mathrm{kin}}$, but are far from these values in the MI. The lower parts show the occupation probabilities for eigenstates after a single spontaneous emission.

resulting wave function is in the ground state (Fig. 3), where we plot occupation probabilities $\left|c_{\alpha}\right|^{2}$ and expectation values of the kinetic energy $\left\langle E_{\alpha}\left|\hat{E}^{\mathrm{kin}}\right| E_{\alpha}\right\rangle$ in the lowest 1000 energy eigenstates $\left|E_{\alpha}\right\rangle$. We find that $E^{\text {kin }}$ grows essentially linearly as a function of $E_{\alpha}$, even for $U / J \sim 3$ near the phase transition, and that these values coincide with $E_{\mathrm{eq}}^{\mathrm{kin}}$ from Boltzmann distributions with corresponding mean energies $E_{\alpha}$. Therefore, a state with $\left|c_{\alpha}\right|^{2}$ leading to an energy expectation $\langle E\rangle$ will approximately have the same kinetic energy as $E_{\mathrm{eq}}^{\mathrm{kin}}$ with mean energy $\langle E\rangle$. Thus, also the long time average $\left\langle E^{\text {kin }}\right\rangle \rightarrow \sum_{\alpha}\left|c_{\alpha}\right|^{2}\left\langle E_{\alpha}\left|\hat{E}^{\text {kin }}\right| E_{\alpha}\right\rangle$ [5] will correspond to $E_{\text {eq }}^{\text {kin }}$ for the corresponding $\langle E\rangle$. As soon as we enter the MI phase, between $U / J \approx 3$ and 3.8 , there is a qualitative change in the distribution of $\left\langle E_{\alpha}\left|\hat{E}^{\text {kin }}\right| E_{\alpha}\right\rangle$, as depicted in Fig. 3, after which we cannot expect to obtain thermal values. In the deep MI, $\left\langle E_{\alpha}\left|\hat{E}^{\mathrm{kin}}\right| E_{\alpha}\right\rangle$ are far from $E_{\mathrm{eq}}^{\mathrm{kin}}$, and correspond to excitations of doublon-holon pairs. In this limit, the system will relax over time to a generalized Gibbs ensemble.

Proposed experimental measurement. We now consider a specific experimental setup in which these effects could be observed. It is important to consider multiple spontaneous emission events, both because of the difficulty of restricting to a single event, and in order to make the change in the momentum distribution sufficiently large to measure. As depicted in Fig. 4(a), we consider a situation in which the background scattering rate is low, and then a moderate scattering rate is induced for a short time $t=1 / J$ (e.g., via a weak beam with near-resonant light). We then switch this off, and observe how the system thermalizes over a time scale of $t \sim 5 / J$. We compute the dynamics of this process by combining t-DMRG methods with quantum trajectory techniques [41], which after a stochastic average allow us to determine the many-body dynamics from the master equation (see Supplemental Material [36]).

In Fig. 4(a), we plot $E^{\text {kin }}$ and $E_{\mathrm{eq}}^{\mathrm{kin}}$ as a function of time. As expected from our single-event calculations, the $E^{\text {kin }}$ increases much faster than would be expected from a thermal distribution with the same increase in total energy 

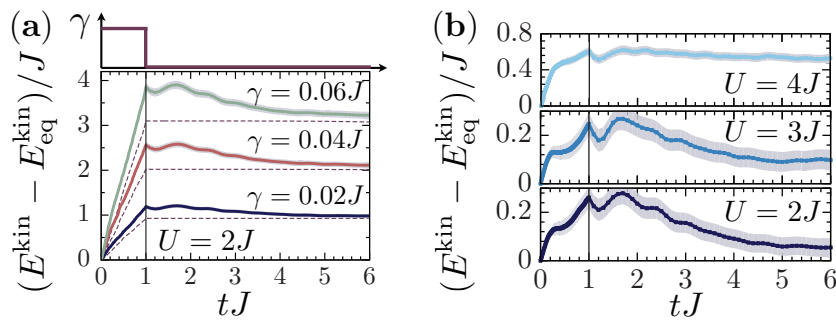

FIG. 4. (Color online) Quantum trajectory simulations for heating with spontaneous emission rate $\gamma$, which is switched on for a time $t=1 / J$, as illustrated above (a). $M=N=48$, and the standard error of the mean is given as the shaded area. (a) The increase in kinetic energy during the heating and the subsequent relaxation for $\gamma=0.02,0.04,0.06$. For superfluid initial states, the kinetic energy relaxes to the equilibrium value (QMC calculations, dashed lines). For a Mott insulating initial state, on the same time scale, the energy does not thermalize. This can be seen in (b), where we plot the difference between the actual kinetic energy and the equilibrium energy (t-DMRG results, $D=256, d_{l}=6,500$ trajectories).

(dashed line), and this is more pronounced for larger $\gamma$. Note that in the experiment of Ref. [24], $\gamma \approx 0.02 J$. In Fig. 4(b) we plot $E^{\text {kin }}-E_{\mathrm{eq}}^{\mathrm{kin}}$ for different values of $U / J$. We see clearly that as in the case of a single spontaneous emission, the kinetic energy will relax towards the expected equilibrium values in the superfluid regime. Strikingly, this is not the case in the Mott insulator, where the system remains well away from the equilibrium value on the time scales calculated. Note that while here the energy increase is small, as we use parameters where few spontaneous emission events occur to allow quantitative numerical treatments, experiments could work with faster scattering rates or longer excitation time scales. Our predictions are observable via momentum distribution measurements that study relaxation in different parameter regimes. This would be enhanced by a quantitative comparison between experimental measurements and QMC calculations (similar to Ref. [24]).

Conclusions. We showed that for bosons in an optical lattice, a change in the thermalization behavior after spontaneous emissions occurs at the SF-MI transition point. Simple quantities including the kinetic energy and quasimomentum distribution settle rapidly to a steady state. However, while in some cases these values correspond to a thermal distribution, in others the values are demonstrably nonthermal. These findings, presented here for a uniform system, remain valid in the presence of a harmonic trap, as is shown by results presented in the Supplemental Material [36]. The lack of complete thermalization implies that the specific effects on specific many-body states must be considered. The generalization of these results to higher dimensions remains an open question, however, because this is a low-energy quench, we expect also that the thermalization properties will be strongly dependent on the detailed low-energy spectrum.

In some regimes, this may lead to greater robustness of states produced in optical lattices, especially where the energy added in a spontaneous emission event would correspond to temperatures above those required for realization of fragile types of order [22-25]. Because the dynamics must instead be treated as a nonequilibrium situation on a case-by-case basis, much of the interesting order can survive on significant time scales.

Acknowledgments. We thank I. Bloch, D. Boyanovsky, W. Ketterle, S. Langer, H. Pichler, U. Schneider, D. Weiss, and P. Zoller for helpful and motivating discussions. This work was supported in part by AFOSR Grant No. FA9550-13-1-0093, by a grant from the U.S. Army Research Office with funding from the DARPA OLE program. We acknowledge the hospitality of the Aspen Center for Physics, supported by NSF Grant No. PHY-1066293. Computational resources were provided by the Center for Simulation and Modeling at the University of Pittsburgh.
[1] J. P. Gordon and A. Ashkin, Phys. Rev. A 21, 1606 (1980).

[2] J. Dalibard and C. Cohen-Tannoudji, J. Phys. B 18, 1661 (1985).

[3] F. Gerbier and Y. Castin, Phys. Rev. A 82, 013615 (2010).

[4] H. Pichler, A. J. Daley, and P. Zoller, Phys. Rev. A 82, 063605 (2010).

[5] M. Rigol, V. Dunjko, and M. Olshanii, Nature (London) 452, 854 (2008).

[6] M. A. Cazalilla, R. Citro, T. Giamarchi, E. Orignac, and M. Rigol, Rev. Mod. Phys. 83, 1405 (2011).

[7] M. Rigol and M. Srednicki, Phys. Rev. Lett. 108, 110601 (2012).

[8] M. Srednicki, Phys. Rev. E 50, 888 (1994).

[9] T. Kinoshita, T. Wenger, and D. S. Weiss, Nature (London) 440, 900 (2006).

[10] M. Rigol, Phys. Rev. Lett. 103, 100403 (2009).

[11] A. C. Cassidy, C. W. Clark, and M. Rigol, Phys. Rev. Lett. 106, 140405 (2011).

[12] M. Rigol and M. Fitzpatrick, Phys. Rev. A 84, 033640 (2011).

[13] I. Bloch, J. Dalibard, and W. Zwerger, Rev. Mod. Phys. 80, 885 (2008).
[14] G. Vidal, Phys. Rev. Lett. 93, 040502 (2004).

[15] A. J. Daley, C. Kollath, U. Schollwöck, and G. Vidal, J. Stat. Mech.: Theory Exp. (2004) P04005.

[16] S. R. White and A. E. Feiguin, Phys. Rev. Lett. 93, 076401 (2004).

[17] F. Verstraete, V. Murg, and J. I. Cirac, Adv. Phys. 57, 143 (2008).

[18] K. Mølmer, Y. Castin, and J. Dalibard, J. Opt. Soc. Am. B 10, 524 (1993).

[19] C. W. Gardiner and P. Zoller, Quantum Noise (Springer, Berlin, 2005).

[20] H. J. Carmichael, An Open Systems Approach to Quantum Optics (Springer, Berlin, 1993).

[21] D. C. McKay and B. DeMarco, Rep. Prog. Phys. 74, 054401 (2011).

[22] S. Fuchs, E. Gull, L. Pollet, E. Burovski, E. Kozik, T. Pruschke, and M. Troyer, Phys. Rev. Lett. 106, 030401 (2011).

[23] R. Jördens, L. Tarruell, D. Greif, T. Uehlinger, N. Strohmaier, H. Moritz, T. Esslinger, L. De Leo, C. Kollath, A. Georges et al., Phys. Rev. Lett. 104, 180401 (2010). 
[24] S. Trotzky, L. Pollet, F. Gerbier, U. Schnorrberger, I. Bloch, N. V. Prokof'ev, B. Svistunov, and M. Troyer, Nat. Phys. 6, 998 (2010).

[25] T. Esslinger, Annu. Rev. Condens. Matter Phys. 1, 129 (2010).

[26] I. Bloch, J. Dalibard, and S. Nascimbene, Nat. Phys. 8, 267 (2012).

[27] J. I. Cirac and P. Zoller, Nat. Phys. 8, 264 (2012).

[28] N. Strohmaier, D. Greif, R. Jördens, L. Tarruell, H. Moritz, T. Esslinger, R. Sensarma, D. Pekker, E. Altman, and E. Demler, Phys. Rev. Lett. 104, 080401 (2010).

[29] A. R. Kolovsky and A. Buchleitner, Europhys. Lett. 68, 632 (2004).

[30] A. R. Kolovsky, Phys. Rev. Lett. 99, 020401 (2007).

[31] M. Rigol, V. Dunjko, V. Yurovsky, and M. Olshanii, Phys. Rev. Lett. 98, 050405 (2007).

[32] G. Biroli, C. Kollath, and A. M. Läuchli, Phys. Rev. Lett. 105, 250401 (2010).
[33] S. S. Natu, K. R. A. Hazzard, and E. J. Mueller, Phys. Rev. Lett. 106, 125301 (2011).

[34] C.-L. Hung, X. Zhang, N. Gemelke, and C. Chin, Phys. Rev. Lett. 104, 160403 (2010).

[35] W. S. Bakr, A. Peng, M. E. Tai, R. Ma, J. Simon, J. I. Gillen, S. Fölling, L. Pollet, and M. Greiner, Science 329, 547 (2010).

[36] See Supplemental Material at http://link.aps.org/supplemental/ 10.1103/PhysRevA.89.011601 for a derivation of Eq. (1), calculations including effects of a harmonic trap, and details of the numerical quantum trajectory technique.

[37] N. Prokof'ev, B. Svistunov, and I. Tupitsyn, J. Exp. Theor. Phys. 87, 310 (1998).

[38] L. Pollet, K. V. Houcke, and S. M. Rombouts, J. Comput. Phys. 225, 2249 (2007).

[39] L. Pollet, Rep. Prog. Phys. 75, 094501 (2012).

[40] E. Toth and P. B. Blakie, Phys. Rev. A 83, 021601(R) (2011).

[41] A. J. Daley, J. M. Taylor, S. Diehl, M. Baranov, and P. Zoller, Phys. Rev. Lett. 102, 040402 (2009). 